\title{
Do Individual Differences Moderate the Cognitive Benefits of Chewing Gum?
}

\author{
Richard Stephens ${ }^{*}$, Nicola M. J. Edelstyn \\ School of Psychology, Keele University, Keele, Staffordshire, UK. \\ Email: r.stephens@psy.keele.ac.uk \\ Received May $26^{\text {th }}, 2011$; revised July $29^{\text {th }}, 2011$; accepted September $8^{\text {th }}, 2011$.
}

\begin{abstract}
Recent experiments investigating whether chewing gum enhances cognitive performance have shown mixed results and a recent replication failed to reproduce earlier findings. The present experiment aimed to investigate whether participant individual differences underlie the discrepant findings. Therefore, in addition to examining differences in Digit Span and Spatial Span performance across gum and control groups, chronotype, extraversion, habitual tiredness, current stress, current arousal and current thirst were assessed using questionnaires. Task difficulty was also manipulated. While there were no chewing gum effects under standard testing conditions, chewing gum enhanced Digit Span performance in the more difficult dual task condition. Furthermore, Spatial Span performance was improved by chewing gum in introverts but not extraverts and chewing gum was shown to eliminate the negative relationship between thirst and Digit Span performance. In explaining these data it is proposed that chewing gum may act both to reduce stress and to alleviate thirst.
\end{abstract}

Keywords: Chewing Gum, Cognitive Performance, Individual Differences, Introversion, Thirst, Stress

\section{Introduction}

Around 90 years ago, when chewing gum sales began to take-off in North America, there were a variety of views pertaining to whether gum was an altogether suitable mass-consumer product. Detractors considered chewing gum to be socially distasteful, even plebian, and unsightly. On the other hand it was claimed in advertisements that chewing gum relieved tension, relaxed the nerves and muscles, aided fatigue, quenched thirst, benefited digestion and slowed tooth decay (Robinson, 2004; see Figure 1 for an example). Nevertheless, the 20th Century saw only one attempt to substantiate the claimed psychological benefits of chewing gum; a study carried out at Columbia University noted a positive correlation between gum chewing and mental task performance (Hollingworth, 1939). More recently, positron emission tomography imaging data has shown a possible mechanism by which chewing could lead to enhanced cognitive performance: increased blood flow in brain regions associated with motor function during chewing (Momose, Nishikawa, Watanabe, Sasaki et al., 1997). Following this discovery, the early part of the 21 st Century saw a resurgence of interest in the cognitive performance effects of chewing gum.

Since 2002 there have been 13 published experiments investigating whether chewing gum benefits young adults' cognitive performance (Wilkinson, Scholey, \& Wesnes, 2002; Baker et al., 2004, experiment 1; Tucha, Mecklinger, Maier, Hammerl, \& Lange, 2004, experiments 1 and 2; Stephens \& Tunney, 2004; Miles \& Johnson, 2007, experiments 1 and 2; Johnson \& Miles, 2007; Johnson \& Miles, 2008 experiment 1; Allen, Norman, \& Katz, 2008; Smith, 2009a; Smith, 2009b; Smith, 2010). These experiments have shown mixed results. So, for example, on the one hand Wilkinson et al. (2002) found that chewing gum enhanced immediate word recall, delayed word recall, spatial working memory and numeric working memory, with some results replicated by Stephens \& Tunney (2004), and Smith (2010) showed improved focused attention and faster reaction time. In the meantime Baker et al. (2004) reported context de-

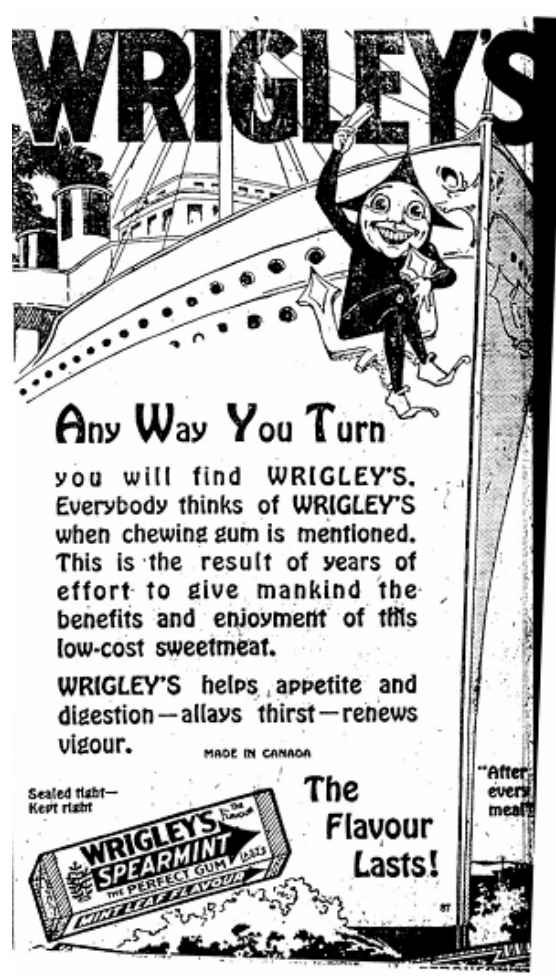

Figure 1.

A 1919 newspaper advertisement for Wrigley's Chewing Gum. Source: London Free Press, 6 January 1919, p. 6. Reproduced from Robinson (2004).

pendent memory effects where the context was set by whether or not participants chewed gum. On the other hand, Tucha et al. (2004) found chewing gum did not enhance participants' immediate and delayed word recall and differentially affected aspects of attention, enhancing sustained attention, showing no effect for divided attention, selective attention, visual scanning 
and vigilance, and showing decrements in tonic alertness, phasic alertness and flexibility. In addition, recent studies have found no memory enhancement from chewing gum (Smith, 2009a; Smith, 2010), no enhanced learning (Allen, Norman, \& Katz, 2008), no improvement in attentional performance (Smith, $2009 \mathrm{~b}$ ) and have not reproduced the context-dependent memory effects that had been observed earlier (Johnson \& Miles, 2007; Miles \& Johnson, 2007; Johnson \& Miles, 2008).

There is no clear pattern to elucidate why findings have been discrepant across experiments. Glucose is known to improve cognitive performance (Sünram-Lea, Foster, Durlach, \& Perez, 2002) and many chewing gum brands include glucose as an ingredient. However, with the exception of Smith (2010), all the above experiments used sugar-free gum, so ruling out glucose as an explanatory factor. While all the experiments employed young adult participants there was some variation in gender distribution across experiments. However, analysis reveals no link between gender distribution and cognitive performance. Of seven experiments with approximately similar numbers of males and females (proportion of males $=40 \%$ $-50 \%$ ) three experiments showed enhanced performance (Tucha et al., 2004, experiment 2; Stephens \& Tunney, 2004; Smith, 2010) and four showed no enhancement (Tucha et al., 2004, experiment 1; Johnson \& Miles, 2007; Allen, Norman, \& Katz, 2008; Smith, 2009b). Of the four experiments in which females more obviously outnumbered males (proportion of males = $13 \%-31 \%$ ) one experiment showed enhanced performance (Baker et al., 2004, experiment 1) and the others showed no enhancement (Miles \& Johnson, 2007, experiments 1 and 2; Johnson \& Miles, 2008 experiment 1). The gender distribution was not stated in two experiments (Wilkinson et al., 2002; Smith, 2009b).

Analytic power also does not explain the pattern of effects in these experiments. Assuming constant effect sizes across studies (which seems reasonable since all the studies are concerned with chewing gum effects on cognition), and ignoring the study that used sugared gum (Smith, 2010), the most highly powered experiments were those conducted by Tucha et al. (experiments 1 and $2 ; n=58$ in a within-subjects design). However, only experiment 2 showed a performance enhancing effect of chewing gum, and that was in only one of several measures of attention employed. On the other hand, Baker et al. experiment 1 appears to have the weakest power ( $n=23$ in a between-subjects design) and yet showed clear context memory effects of gum. It is particularly troubling that in an exact replication Johnson and Miles (2007) were unable to demonstrate improved recall following chewing gum at first presentation and retrieval as reported by Baker et al (2004; experiment 1).

The absence of any clear methodological reason why some studies showed effects while others did not together with the failures to replicate described above lead us to consider whether the different experimental findings reflect something about the condition of the participants employed across studies. For example, perhaps in some of the previous studies participants were experiencing mild sleep deprivation and chewing gum helped reverse the deficit associated with this. This is analogous with research designs aiming to assess the extent to which caffeine and other drugs offset cognitive deficits arising due to sleep deprivation (e.g. Wesensten, Belenky, Kautz, Thorne, Reichardt, \& Balkin, 2002). Indeed, Hodoba (1999) found that participants who stayed up all night chewing gum reported feeling less tired than controls who stayed up all night without chewing gum. It is not routine in laboratory-based psychology experiments for data concerning background factors such as fatigue to be collected and reported, and indeed such data were not reported in the chewing gum studies reviewed above. In the majority of the chewing gum experiments to date the participants were drawn from the undergraduate student population. Gill (2002) reports that up to half of male students may exceed sensible weekly alcohol guidelines and take part in binge drinking. Therefore, there is undoubtedly scope within this group for participants to attend the laboratory inadequately rested, or in some otherwise sub-optimal state.

Therefore, in the present study in addition to examining differences in cognitive performance across gum and control groups, we also used several questionnaires to assess dimensions relating to individual differences in participants. This is a novel approach in the research on chewing-gum effects on cognitive performance. Three variables pertinent to tiredness were assessed-chronotype (whether a person is most productive in the morning or the evening hours) was assessed using the Morningness-Eveningness Scale (Horne \& Ostberg, 1976); habitual tiredness (as opposed to current level of tiredness) was assessed using the Epworth Sleepiness Scale (Johns, 1991); and current arousal level was assessed using the Stress and Arousal Checklist (Cox \& Mackay, 1985). This was on the basis that chewing gum-mediated cognitive enhancement would be more likely to be present in participants who were tired or fatigued relative to those that were not so affected. In addition extraversion was assessed using the Eysenck Personality Inventory (Eysenck \& Eysenck, 1967) as there is a body of work suggesting that introverts (individuals scoring at the low end of the extraversion scale) are chronically more highly aroused compared with extraverts (Matthews, Davies, Westerman, \& Stammers, 2004). Two additional factors known to affect cognitive performance were also assessed. These were current stress level, assessed using the Stress and Arousal Checklist (Cox \& Mackay, 1985), and current thirst level assessed using a single 9-response Likert scale following the method reported by Rogers, Kainth $\&$ Smit (2001). The latter variable was included following a suggestion made during the 2007 British and Feeding Drinking Society symposium on chewing gum effects on cognition (Stephens \& Tunney, 2008) that gum may exert its cognitive enhancement effects by alleviating thirst. Certainly, data exist linking thirst with cognitive decrement (e.g. Rogers, Kainth, \& Smit, 2001).

A further manipulation was to vary task difficulty. In their null-effect study of chewing gum effects on learning in dental students, Allen et al. (2008) questioned whether chewing gum may be more likely to benefit "less academically accomplished" individuals (p. 106) compared with high-performing students. What they are implying here is that a law of diminishing returns acts in relation to the potential for enhancement of cognitive performance such that enhancement is less likely to occur the higher the performance baseline. Such a concept seems similar to Wilder's law of initial value, defined as "the higher the initial value, the smaller the response of functionraising, the larger the response to function-depressing stimuli" (as cited in Jin, 1992, p. 176). Although usually applied in psychophysiological research, Wilder's law of initial value captures the concept that it becomes more difficult to improve performance the higher the performance baseline. If this assumption is correct, then any performance benefit of chewing gum would be relatively smaller in individuals performing near optimal level compared with those performing less well. Therefore, to create conditions under which the level of performance would be more likely to dip below optimal, we increased cognitive task difficulty by adding the secondary task of concurrently 
counting the incidence of specified events occurring during certain trials.

The cognitive tests employed assessed verbal and spatial working memory. These tests were selected on the basis that they were quick and easy to administer, and because working memory has been shown to be enhanced by chewing gum (Wilkinson, Scholey, \& Wesnes, 2002; Stephens \& Tunney, 2004). We did not expect to see cognitive enhancement effects in participants chewing gum compared with those not chewing under the standard testing condition. However, cognitive enhancement with chewing gum was hypothesised in the dual task (difficult) condition. Furthermore, cognitive enhancement with chewing gum was hypothesised regardless of task difficulty for individuals reporting any of: higher levels of evening typeness, higher levels of tiredness, lower levels of arousal, higher levels of extraversion, higher levels of stress and/or higher levels of thirst.

\section{Methods}

\section{Participants}

Participants, 29 females and 17 males, of mean age 21.04 years (SD 1.49), were recruited through an opportunity sample of Keele University students.

\section{Materials}

A tape-recorded version of the Digit Span test was used to assess verbal working memory (Wechsler, 1981) and the Spatial Span test was used to assess visuospatial working memory (Wechsler, 1999). In addition to the standard test forms the tests were also performed under a dual-task condition with the aim of increasing task difficulty. For Digit Span participants were required to report the number of occurrences of a brief tone that sounded occasionally during the number sequence presentation ( 0 - 3 occasions per trial). Participants had to report accurately both the number of tones sounded and the digit sequence in order to "pass" each trial. For Spatial Span the investigator occasionally used two fingers to point at the board rather than one (0 - 3 occasions per trial). Participants were asked to report the number of occurrences of two fingered pointing in each trial as well as the correct sequence of blocks; both had to be accurate to "pass" each trial.

Several questionnaires were used. The Morningness-Eveningness Scale (Horne \& Ostberg, 1976) was used to assess chronotype; lower scores are indicative of morning-typeness. The Eysenck Personality Inventory (Eysenck \& Eysenck, 1967) was used to assess the personality dimension extraversion; scores on this questionnaire form a continuum with lower scores indicating introversion and higher scores indicating extraversion. The Epworth Sleepiness Scale (Johns, 1991) was employed to assess habitual tiredness; higher scores are indicative of greater habitual tiredness. The Stress and Arousal Check List (Cox \& Mackay, 1985) was used to assess the extent to which participants were feeling i) stressed and ii) aroused at the time of questionnaire completion; higher scores are indicative of higher current levels for each variable. Finally a single item 9-point Likert scale was used to assess participants' current thirst (Rogers et al., 2001); a higher score is indicative of greater thirst. The chewing gum employed was Wrigley's Extra Sugar-free Cool Breeze $e^{T M}$.

\section{Design}

A mixed measures general linear design was employed, with one between-subjects categorical predictor variable, gum (gum vs. no gum); one within-subjects categorical predictor variable, task difficulty (standard vs. dual task); and one continuous between-subjects predictor variable, questionnaire score (one of: chronotype; extraversion; habitual tiredness; current stress; current arousal; and current thirst). The dependent variables were the Digit Span and Spatial Span scores. Participants were randomly assigned to the gum and no gum conditions. The order in which participants encountered the standard and dual task conditions was randomised. Two alternative forms of the standard and dual task Digit Span and Spatial Span tests were employed; test form was randomised across conditions.

\section{Procedure}

Keele University School of Psychology Research Ethics Committee approved the study. Participant consent was a prerequisite for participation. For participant recruitment purposes the research was described as a cognitive performance study. Testing occurred between 9.00 am and $2.00 \mathrm{pm}$. All participants completed the questionnaires first and the tests of working memory next. Participants in the "gum" condition commenced chewing a single piece of gum immediately prior to the cognitive testing and continued chewing throughout testing. After completing the cognitive tests the participants were thanked, debriefed and offered a free pack of chewing gum or sweets.

\section{Results}

Participants' questionnaire and working memory test scores in each of the experimental conditions are shown in (Table 1), below.

There were four analytic stages. In the first stage only data from the standard Digit Span and Spatial Span tests were analysed using one-way ANOVAs comparing performance of the

Table 1.

Means and standard deviations of the questionnaire and cognitive test scores in the gum and control conditions.

\begin{tabular}{|c|c|c|c|c|}
\hline & \multicolumn{2}{|c|}{$\operatorname{Gum}(n=23)$} & \multicolumn{2}{|c|}{ Control $(n=23)$} \\
\hline & Mean & SD & Mean & SD \\
\hline Age & 20.78 & 0.85 & 21.30 & 1.92 \\
\hline $\begin{array}{c}\text { Chronotype } \\
(\text { Maximum Score }=30)\end{array}$ & 14.00 & 4.36 & 18.22 & 4.94 \\
\hline $\begin{array}{c}\text { Extraversion } \\
(\text { Maximum Score }=24)\end{array}$ & 13.48 & 4.44 & 12.48 & 4.89 \\
\hline $\begin{array}{c}\text { Habitual Tiredness } \\
\text { (Maximum Score }=24)\end{array}$ & 8.70 & 3.83 & 7.22 & 2.59 \\
\hline $\begin{array}{c}\text { Current Stress } \\
(\text { Maximum Score }=18)\end{array}$ & 4.52 & 4.14 & 2.52 & 2.27 \\
\hline $\begin{array}{c}\text { Current Arousal } \\
(\text { Maximum Score }=12)\end{array}$ & 8.00 & 2.98 & 9.04 & 2.18 \\
\hline $\begin{array}{c}\text { Current Thirst } \\
(\text { Maximum Score }=9)\end{array}$ & 6.22 & 1.81 & 6.09 & 2.00 \\
\hline $\begin{array}{l}\text { Standard WAIS-R Digit Span } \\
\quad(\text { Maximum Score }=28)\end{array}$ & 14.91 & 3.26 & 14.65 & 4.28 \\
\hline $\begin{array}{l}\text { Dual Task Digit Span } \\
\text { (Maximum Score }=28 \text { ) }\end{array}$ & 14.26 & 3.31 & 12.57 & 4.52 \\
\hline $\begin{array}{l}\text { Standard WMS-III Spatial Span } \\
\quad(\text { Maximum Score }=28)\end{array}$ & 15.57 & 3.09 & 14.70 & 2.90 \\
\hline $\begin{array}{l}\text { Dual Task Spatial Span } \\
(\text { Maximum Score }=28)\end{array}$ & 14.13 & 3.43 & 12.65 & 2.69 \\
\hline
\end{tabular}


chewing group with the control group. In the second stage mixed $2 \times 2$ ANOVAs were computed examining the effects on Digit Span and Spatial Span test performance of the between subjects factor gum (gum vs. no gum), the within subjects factor task difficulty (standard vs. dual task) and the interaction of these factors. In the third analytic stage a series of mixed measures general linear models were applied. These included the between-subjects categorical predictor variable, gum (gum vs. no gum), the within-subjects categorical predictor variable, task difficulty (standard vs. dual task), and a continuous betweensubjects predictor variable, questionnaire score. Two sets of these analyses were applied. In the first set the dependent variable was Digit Span test score, while in the second set the dependent variable was Spatial Span test score. Within each set six general linear models were computed. Each general linear model included the categorical predictors described above, but each general linear model included one of the following six questionnaire-derived continuous predictor variables: chronotype; extraversion; habitual tiredness; current stress; current arousal; and current thirst. Each general linear model included the three-way interaction (gum by task difficulty by questionnaire score), the three two-way interactions (gum by task difficulty; gum by questionnaire score; and task difficulty by questionnaire score) and the three main effects (gum; task difficulty; and questionnaire score). In the fourth analytic stage bivariate correlations among the six questionnaire-derived continuous predictor variables were computed, in order to facilitate the interpretation of the third phase analyses. All analyses were carried using SPSS v16.

\section{First Stage Analyses-Gum vs. Control on the Standard Cognitive Tests}

There was no significant difference in Digit Span performance between the gum and control groups, $\mathrm{F}(1,44)<1$, partial eta squared $=0.001$, and nor was there a significant difference in Spatial Span performance between the gum and control groups, $\mathrm{F}(1,44)<1$, partial eta squared $=0.022$.

\section{Second Stage Analyses-Gum vs. Control on the Standard and Dual Task Cognitive Tests}

For Digit Span, there was a significant gum by difficulty interaction, $\mathrm{F}(1,44)=4.844, \mathrm{p}=0.033$, partial eta squared $=$ 0.099 . This interaction is depicted in Figure 2. Analytic comparisons showed that performance in the more difficult dual task condition was improved in the gum group compared with controls, $\mathrm{F}(1,44)=13.535, \mathrm{p}=0.001$, but there was no performance difference between the gum and control groups on the standard test, $\mathrm{F}(1,44)<1$.

For Spatial Span, the gum by difficulty interaction was not significant, $F(1,44)<1$, partial eta squared $=0.018$, and neither was the main effect of gum, $\mathrm{F}(1,44)=2.009, \mathrm{p}=0.163$, partial eta squared $=0.044$. However there was a significant main effect of difficulty, $\mathrm{F}(1,44)=25.873, \mathrm{p}<0.001$, partial eta squared $=0.370$, such that scores in the more difficult dual task condition were reduced compared with the standard Spatial Span test.

\section{Third Stage Analysis-Gum vs. Control on the Standard and Dual Task Cognitive Tests Taking into Account Individual Differences}

There were no significant three-way interaction effects. A significant gum by extraversion interaction predicted Spatial Span performance, $\mathrm{F}(1,42)=6.578, \mathrm{p}=0.014$, partial eta

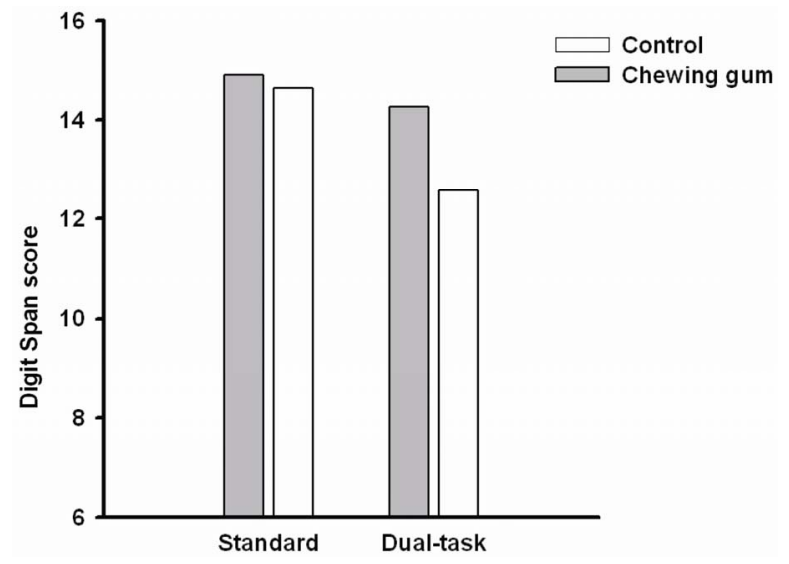

Figure 2.

Digit span performance as a function of task difficulty (standard vs. dual task condition) in the chewing gum and control conditions.

squared $=0.135$. This interaction is illustrated in Figure 3. Underlying this interaction was a non-significant regression of extraversion on Spatial Span in the controls, $r=0.107, \mathrm{~F}(1,22)<1$, partial eta squared $=0.041$, whereas the regression of extraversion on Spatial Span in the chewing gum group was significant, $r$ $=-0.333, \mathrm{~F}(1,22)=6.519, \mathrm{p}=0.019$, partial eta squared $=0.237$.

A significant gum by current thirst interaction predicted Digit Span performance, $\mathrm{F}(1,42)=4.642, \mathrm{p}=0.037$, partial eta squared $=$ 0.100 . This interaction is illustrated in (Figure 4). Underlying this interaction was a significant regression of current thirst on Digit Span in the controls, $\mathrm{r}=-1.135, \mathrm{~F}(1,21)=7.998, \mathrm{p}=0.010$, partial eta squared $=0.276$, whereas the regression of current thirst on Digit Span in the chewing gum group was not significant, $r=0.043$, $\mathrm{F}(1,21)<1$, partial eta squared $=0.001$.

There were no other significant two-way interaction effects. The only significant main effect of chewing gum, which occurred on Spatial Span performance in the general linear model including extraversion, is superseded by the interaction of gum and extraversion described above. In the general linear models predicting Spatial Span performance including the continuous variable predictors, chronotype, habitual tiredness, and current stress, there were main effects of task difficulty, $F(1,42) \geq$ $4.285, p \leq 0.045$, partial eta-squared $\geq 0.093$. In all cases performance was reduced in the dual task condition. There was a single main effect of task difficulty on Digit Span performance

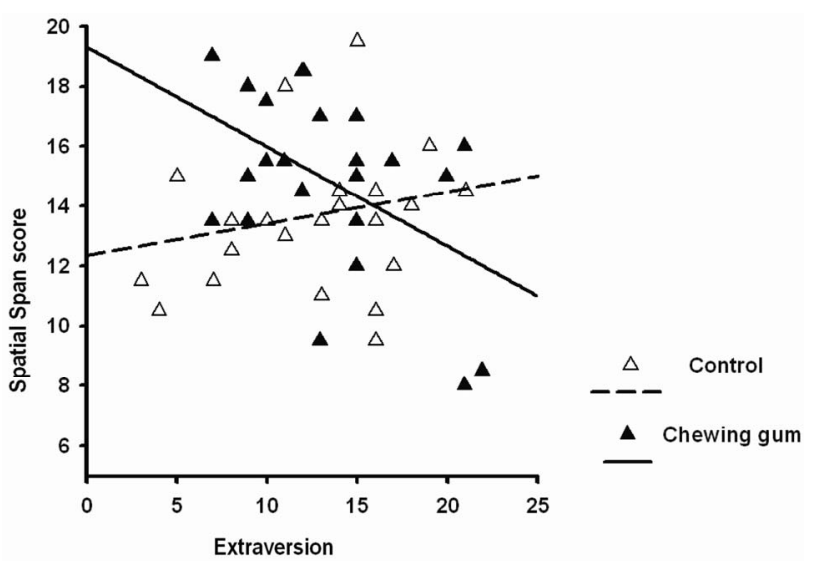

Figure 3.

Spatial span performance as a function of extraversion in the chewing gum and control conditions. 


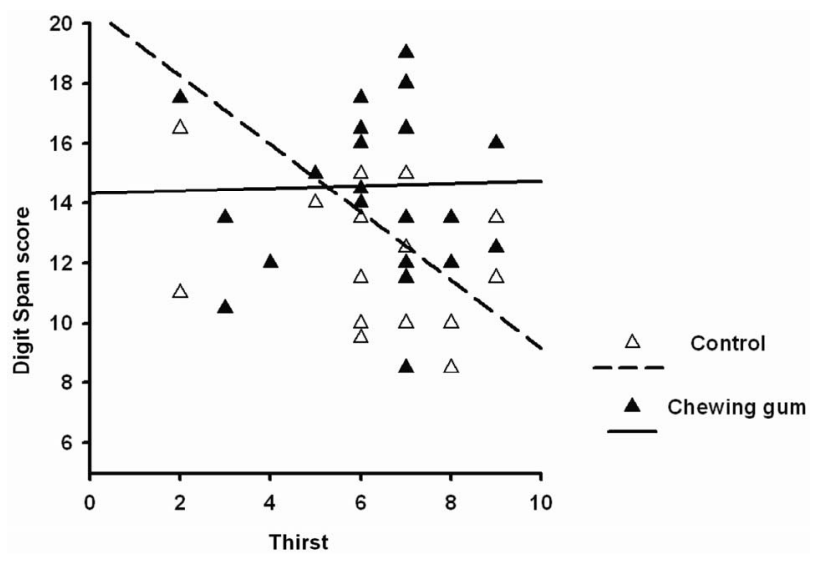

Figure 4.

Digit span performance as a function of thirst in the chewing gum and control conditions.

such that performance was reduced in the dual task condition in Underlying this interaction was a non-significant regression of the general linear model including current stress as a predictor, $\mathrm{F}(1,42)=6.593, \mathrm{p}=0.014$, partial eta-squared $=0.136$.

\section{Fourth Stage Analysis-Bivariate Correlations among the Six Questionnaire-Derived Continuous Predictor Variables}

Pearson product-moment correlation coefficients were computed for all bivariate combinations of the six questionnairederived continuous predictor variables: chronotype; extraversion; habitual tiredness; current stress; current arousal; and current thirst. The results are summarised in Table 2. The only significant correlation was the negative relationship between current stress and current arousal.

\section{Discussion}

Chewing gum did not enhance working memory performance in the analyses including only the standard Digit Span and Spatial Span tests. In this sense the present study has failed to replicate our earlier findings (Stephens \& Tunney, 2004) and those of other researchers (Wilkinson et al., 2002; Baker et al., 2004, experiment 1; Tucha et al., 2004, experiment 2). However, the absence of enhanced cognitive performance brought about by chewing gum is consistent with other research (Tucha et al., 2004, experiment 1; Miles \& Johnson, 2007; Johnson \& Miles, 2007; Allen, Norman, \& Katz, 2008; Johnson \& Miles, 2008; Smith, 2009a; Smith, 2009b). Nevertheless, chewing gum was shown to be beneficial to performance compared with not chewing on the Digit Span test when a secondary task of counting sounded tones was concurrently performed. We employed this more difficult dual task condition to induce sub-optimal performance on the assumption that a law of initial value (Jin, 1992) was in operation such that chewing gum would be more likely to improve cognitive performance when it was initially sub-optimal. These data are consistent with the interpretation that chewing gum is most likely to improve cognitive performance in individuals who are performing at a sub-optimal level.

The most intriguing findings reported here occurred in the analyses incorporating questionnaire-derived variables reflecting individual differences. For thirst, the controls showed decreased Digit Span performance with increasing levels of thirst, as predicted by some of the literature on effects of hydration and dehydration on cognitive performance (e.g. Rogers, Kainth, $\&$ Smit, 2001). However, in the chewing gum condition no such decline in performance was present. It has been suggested that chewing gum may be of benefit to cognitive performance via reducing feelings of thirst, and indeed some of the earliest recorded chewing gum marketing materials (see Figure 1) alluded to the thirst-quenching properties of chewing gum (Robinson, 2004). Previous research found that using sugar-free chewing gum six times daily for 2 weeks reduced self-reported thirst in 137 haemodialysis patients, mediated by stimulation of saliva flow (Bots, Brand, Veerman, Korevaar et al., 2005). The findings of the present study are consistent with a role for chewing gum in alleviating thirst.

Furthermore, extraversion was a significant predictor of Spatial Span test performance in the chewing gum group, such that better performance was observed in more introverted individuals. However, extraversion did not predict Spatial Span test performance in the control group. This runs counter to our initial hypothesis. Previous research has found that extraverts tend to perform well under more arousing conditions, and that introverts perform better under less arousing conditions (Matthews, Davies, Westerman, \& Stammers, 2004) and on this basis we predicted that chewing gum would be likely to overarouse introverts leading to reduced cognitive performance. On the other hand, we had predicted that chewing gum would be more likely to optimally arouse extraverts, leading to performance benefits in extraverts. Recent research suggests that stress theory may be a parsimonious way to explain these findings. A modest correlation $(r=0.44)$ was found between anxiety and introversion in a survey of 441 adults in Finland (Jylhä \& Isometsä, 2006). Furthermore, chewing gum was shown to reduce self-rated stress and salivary cortisol in 40 young adults during completion of a stressful cognitive test battery (Scholey,

Table 2.

Pearson product-moment correlation coefficients for all bivariate combinations of the six questionnaire-derived continuous predictor variables. $N=$ 46 in all cases; $p>0.05$ in all cases except for stress-arousal.

\begin{tabular}{cccccc}
\hline & Chronotype & Extraversion & Habitual tiredness & Current Stress & Current Arousal \\
\hline Extraversion & -0.014 & & & \\
Habitual Tiredness & -0.121 & 0.252 & & \\
Current Stress & -0.211 & 0.064 & 0.039 & $-0.496^{*}$ & 0.247 \\
Current Arousal & 0.007 & -0.119 & -0.150 & 0.125 & -0.123 \\
Current Thirst & 0.238 & -0.253 & & \\
\hline
\end{tabular}


Haskell, Robertson, Kennedy, Milne, \& Wetherell, 2009). Perhaps introverts experienced more stress during the cognitive testing procedure than extraverts and this stress was alleviated by chewing gum. Further research should examine links between introversion, cognitive testing and stress.

In 2004 when we published our first experiment on chewing gum and cognitive performance (Stephens \& Tunney, 2004) several studies including our own showed beneficial effects while only one study pointed to the absence of cognitive benefits (Tucha et al., 2004), which we interpreted as anomalous. Consequently, our suggested mechanistic models for chewing gum effects on cognition were somewhat simplistic. At that time we proposed that gum chewing exerted cognitive benefits either via activity-induced adrenergic arousal, or via increased cerebral blood flow as a by-product of mastication. However, given the absence of any link between chewing gum mediated cognitive benefits and initial low arousal in the present study, the former mechanism appears less feasible, although Smith (2009a) argues that chewing gum has a robust alerting effect, and so perhaps our study lacked sufficient sensitivity to properly assess arousal. Furthermore, an effect mediated solely by cerebral blood flow seems unlikely given that the present data indicate that chewing only produces cognitive benefits under certain circumstances. However, a mechanism whereby enhanced cognition occurs due to a reduction in stress brought about by cardiovascular changes consequent to chewing is a possibility, and follows the suggestion of a link between stress relief and cardiovascular changes brought about by chewing made by Scholey et al. (2009).

Allied to this it possible that the repetitive nature of chewing, producing stereotypical movement acts to soothe in the same way that a baby is soothed by rocking. Indeed, a central tenet of the first investigation of the psychological effects of chewing gum was the idea that the "collateral motor automitism" involved in sustained mastication results in a lowering of tension (Hollingworth, 1939). Given the individual differences noted in the present study, stress alleviation may be one of two key factors in determining when chewing gum will lead to cognitive benefits. The other key factor is that chewing gum appears to alleviate thirst, and consequently may attenuate the negative cognitive performance effects of thirst.

In conclusion, the present research has replicated the null cognitive effects observed in several recent chewing gum experiments. However, the study also moves chewing gum-cognition research forward by identifying conditions and participant characteristics (individual differences) where chewing gum is more likely to confer cognitive benefits. Our findings indicate that chewing may benefit cognitive performance where people feel thirsty, in introverts (but not extraverts) and where mental performance is sub-optimal. While the precise mechanism for the effect remains unknown, cognitive benefits may occur as a consequence of chewing gum-mediated alleviation of stress and of thirst.

\section{References}

Allen, K. L., Norman, R. G., \& Katz, R. V. (2008). The effect of chewing gum on learning as measured by test performance. Nutrition Bulletin, 33, 102-107. doi:10.1111/j.1467-3010.2008.00697.x

Baker, J. R., Benzance, J. B., Zeilaby, E., \& Aggleton, J. P. (2004). Chewing gum can produce context-dependent effects upon memory. Appetite, 43, 207-210. doi:10.1016/j.appet.2004.06.004

Bots, C. P., Brand, H. S., Veerman, E. C. I., Korevaar, J. C., Valentijn-Benz, M., Bezemer, P. D., Valentijn, R. M., Vos, P. F., Bijisma,
J. A., Wee, P. M., Van Amerongen, B. M., \& Amerongen A. V. N. (2005). Chewing gum and a saliva substitute alleviate thirst and xerostomia in patients on haemodialysis. Nephrology Dialysis Transplantation, 20, 578-584. doi:10.1093/ndt/gfh675

Cox, T., \& Mackay, C. (1985). The measurement of self-reported stress and arousal. British Journal of Psychology, 76, 183-186. doi:10.1111/j.2044-8295.1985.tb01941.X

Eysenck, H. J., \& Eysenck, S. G. B. (1965). The eysenck personality inventory. British Journal of Psychology, 14, 140. doi: $10.2307 / 3119050$

Gill, J. S. (2002). Review: Reported levels of alcohol consumption and binge drinking within the UK undergraduate student population over the last 25 years. Alcohol and Alcoholism, 37, 109-120. doi:10.1093/alcalc/37.2.109

Hodoba, D. (1999). Chewing can relieve sleepiness in a night of sleep deprivation. Journal of Sleep Research, 2, 101-105.

Hollingworth, H. L. (1939). Chewing as a technique of relaxation. Science, 90, 385-387. doi:10.1126/science.90.2339.385

Horne, J. A., \& Ostberg, O. (1976). A self assessment questionnaire to determine morningness-eveningness in human circadian rhythms. International Journal of Chronobiology, 4, 97-110.

Jin, P. (1992). Toward a reconceptualization of the law of initial value. Psych Bull, 111, 176-184. doi:10.1037/0033-2909.111.1.176

Johns, M. W. (1991). A new method for measuring daytime sleepiness: The epworth sleepiness scale. Sleep, 14, 540-545.

Johnson, A., \& Miles, C. (2007). Evidence against memorial facilitation and context-dependent memory effects through the chewing of gum. Appetite, 48, 394-396. doi:10.1016/j.appet.2006.10.003

Johnson, A., \& Miles, C. (2008). Chewing gum and context-dependent memory: The independent roles of chewing gum and mint flavour. British Journal of Psychology, 99, 293-306. doi: $10.1348 / 000712607 X 228474$

Jylhä, P., \& Isometsä, E. (2006). The relationship of neuroticism and extraversion to symptoms of anxiety and depression in the general population. Depression and Anxiety, 23, 281-289. doi:10.1002/da.20167

Matthews, G., Davies, D. R., Westerman, S. J., \& Stammers, R. B. (2004). Human performance: Cognition, stress and individual differences. Hove: Psychology Press.

Miles, C., \& Johnson, A. J. (2007). Chewing gum and context-dependent memory effects: A re-examination. Appetite, 48, 154-158. doi:10.1016/j.appet.2006.07.082

Momose, I., Nishikawa, J., Watanabe, T., Sasaki, Y., Senda, M., Kubota, K., Sato, Y., Funakoshi, M., \& Minakuchi, S. (1997). Effect of mastication on regional cerebral blood flow in humans examined by positron-emission tomography with 15O-labelled water and magnetic resonance imaging. Archives of Oral Biology, 42, 57-61. doi:10.1016/S0003-9969(96)00081-7

Robinson, D. (2004). Marketing gum, making meanings: Wrigley in North America, 1890-1930. Enterprise \& Society, 5, 4-44. doi:10.1093/es/khh002

Rogers, P. J., Kainth, A., \& Smit, H. J. (2001). A drink of water can improve or impair mental function depending on small differences in thirst. Appetite, 36, 57-58. doi:10.1006/appe.2000.0374

Scholey, A., Haskell, C., Robertson, B., Kennedy, D., Milne, A., \& Wetherell, M. (2009). Chewing gum alleviates negative mood and reduces cortisol during acute laboratory psychological stress. Physiology \& Behavior, 97, 304-312. doi:10.1016/j.physbeh.2009.02.028

Smith, A. (2009a). Effects of chewing gum on mood, learning, memory and performance of an intelligence test. Nutritional Neuroscience, 12, 81-88. doi:10.1179/147683009X423247

Smith, A. (2009b). Effects of caffeine in chewing gum on mood and attention. Human Psychopharmacology: Clinical and Experimental, 24, 239-247. doi:10.1002/hup.1020

Smith, A. (2010). Effects of chewing gum on cognitive function, mood and physiology in stressed and non-stressed volunteers. Nutritional Neuroscience, 13, 7-16. doi:10.1179/147683010X12611460763526

Stephens, R., \& Tunney, R. J. (2004). Role of glucose in chewing gumrelated facilitation of cognitive function. Appetite, 43, 211-213. doi:10.1016/j.appet.2004.07.006

Stephens, R., \& Tunney, R. J. (2008). Role of glucose in chewing gumrelated facilitation of cognitive function. Appetite, 50, 566. doi:10.1016/j.appet.2007.09.059 
Sünram-Lea, S. I., Foster, J. K., Durlach, P., \& Perez, C. (2002). Investigation into the significance of task difficulty and divided allocation of resources on the glucose memory facilitation effect. Psychopharmacology (Berl), 160, 387-397. doi:10.1007/s00213-001-0987-9

Tucha, O., Mecklinger, L., Maier, K., Hammerl, M., \& Lange, K. W. (2004). Chewing gum differentially affects aspects of attention in healthy subjects. Appetite, 42, 327-329.

doi:10.1016/j.appet.2004.01.003

Wechsler, D. (1981). Manual for the wechsler adult intelligence scale, revised. The Psychological Corporation: San Antonio.
Wechsler, D. (1999). Wechsler memory scale III manual. The Psychological Corporation: New York.

Wesensten, N., Belenky, G., Kautz, M. A., Thorne, D. R., Reichardt, R. M., \& Balkin, T. J. (2002). Maintaining alertness and performance during sleep deprivation: modafinil versus caffeine. Psychopharmacology (Berl), 159, 238-247. doi:10.1007/s002130100916

Wilkinson, L., Scholey, A., \& Wesnes, K. (2002). Chewing gum selectively improves aspects of memory in healthy volunteers. Appetite, 38, 235-236. doi:10.1006/appe.2002.0473 